\title{
Iteratively Decoded Variable Length Space-Time Coded Modulation: Code Construction and Convergence Analysis
}

\author{
Soon Xin Ng, Member, IEEE, Jin Wang, Student Member, IEEE, Meixia Tao, Member, IEEE, \\ Lie-Liang Yang, Senior Member, IEEE, and Lajos Hanzo, Fellow, IEEE
}

\begin{abstract}
An Iteratively Decoded Variable Length Space Time Coded Modulation (VL-STCM-ID) scheme capable of simultaneously providing both coding and iteration gain as well as multiplexing and diversity gain is proposed. Non-binary unity-rate precoders are employed for assisting the iterative decoding of the VL-STCM-ID scheme. The discrete-valued source symbols are first encoded into variable-length codewords that are mapped to the spatial and temporal domains. Then the variable-length codewords are interleaved and fed to the precoder assisted modulator. More explicitly, the proposed VL-STCM-ID arrangement is a jointly designed iteratively decoded scheme combining source coding, channel coding, modulation as well as spatial diversity/multiplexing. As expected, the higher the source correlation, the higher the achievable performance gain of the scheme becomes. Furthermore, the performance of the VLSTCM-ID scheme is about 14.6 dB better than that of the Fixed Length STCM (FL-STCM) benchmarker at a source symbol error ratio of $10^{-4}$.
\end{abstract}

Index Terms - EXIT charts, iterative decoding, MIMO, STTC, VLC.

\section{INTRODUCTION}

$\mathbf{S}$ HANNON'S separation theorem stated that source coding and channel coding is best carried out in isolation [1]. However, this theorem was formulated in the context of potentially infinite-delay, lossless entropy-coding and infinite block length channel coding. In practise, real-time wireless audio/video communications systems do not meet these ideal hypotheses. Explicitly, the source encoded symbols often remain correlated, despite the lossy source encoder's efforts to remove all redundancy. Furthermore, they exhibit unequal error sensitivity. In these circumstances, it is often more efficient to use jointly designed source and channel encoders.

The wireless communication systems of future generations are required to provide reliable transmissions at high data rates in order to offer a variety of multimedia services. Space

Manuscript received September 14, 2005; revised January 8, 2006; accepted March 9, 2006. The associate editor coordinating the review of this paper and approving it for publication was G. Vitetta. This paper was published in part in S. X. Ng, J. Wang, L.-L. Yang, and L. Hanzo: Variable Length Space Time Coded Modulation, Proceedings of IEEE VTC'05 Fall, Dallas, 26-28 of Sep. 2005, pp. 1049-1053.

S. X. Ng, J. Wang, L.-L. Yang, and L. Hanzo are with the School of Electronics and Computer Science, University of Southampton, SO17 1BJ, UK (e-mail: \{sxn,jw02r,lh,lly\}@ecs.soton.ac.uk).

M. Tao is with the Department of Electrical and Computer Engineering, National University of Singapore, 4 Engineering Drive 3, Singapore 117576 (e-mail:mxtao@nus.edu.sg).

Digital Object Identifier 10.1109/TWC.2007.05698 time coding schemes, which employ multiple transmitters and receivers, are among the most efficient techniques designed for providing high data rates by exploiting the high channel capacity potential of Multiple-Input Multiple-Output (MIMO) channels [2], [3]. More explicitly, Bell-lab's LAyered Space Time architecture (BLAST) [4] was designed for providing full-spatial-multiplexing gain, while Space Time Trellis Codes (STTC) [5] were designed for providing full-spatial-diversity gain.

The novel contribution of this paper is that we propose a jointly designed source coding and Space Time Coded Modulation (STCM) scheme, where two dimensional (2D) Variable Length Codes (VLCs) are transmitted by exploiting both the spatial and temporal domains. More specifically, the number of activated transmit antennas equals the number of symbols of the corresponding VLC codeword in the spatial domain, where each VLC codeword is transmitted during a single symbol period. Hence, the transmission frame length is determined by the fixed number of source symbols and therefore the proposed Variable Length STCM (VL-STCM) scheme does not exhibit synchronisation problems and does not require the transmission of side information. Additionally, the associated source correlation is converted into an increased minimum product distance ${ }^{1}$, hence resulting in an increased coding gain. Furthermore, the VL-STCM scheme advocated is capable of providing both multiplexing and diversity gains with the aid of multiple transmit antennas. Practical applications of the proposed scheme are related to the transmission of VLC-based MPEG 2, 3 and 4 encoded video and audio sequences for example. It is also possible to simply pack binary computer-data into VLC-encoded symbols for the sake of their near-capacity transmission.

Relevant work on the joint design of source coding and space-time coding can be found in [6] and [7], where the performance measure is based on the end-to-end analogue source distortion. However, in this paper we assume the presence of a discrete source where the potentially analogue source was quantized/discretized, before it was input to our VL-STCM encoder. Our objective is thus to minimise the error probability of the discrete source symbols. Furthermore, joint detection of conventional one dimensional (1D) VLC and

\footnotetext{
${ }^{1}$ This will be explained in Section III.
} 
STCM has been shown to approach the channel capacity in [8], although the related VLC schemes have to convey explicit side information regarding the total number of VLC encoded bits or symbols per transmission frame.

On the other hand, it was shown in [9] that a binary UnityRate Code (URC) or precoder can be beneficially concatenated with Trellis Coded Modulation (TCM) [10] for the sake of invoking iterative detection and hence for attaining iteration gains. Since VL-STCM also belongs to the TCM family, we further develop the VL-STCM scheme for the sake of attaining additional iteration gains by introducing a novel nonbinary URC between the variable-length space-time encoder and the modulator. The Iteratively Decoded (ID) VL-STCM (VL-STCM-ID) scheme achieves a significant coding/iteration gain over both the non-iterative VL-STCM scheme and the Fixed Length STCM (FL-STCM) benchmarker.

The paper is organised as follows. The overview of the space-time coding technique advocated is given in Section II and the 2D VLC design is outlined in Section III. The description of the proposed VL-STCM and VL-STCM-ID schemes is presented in Sections IV and $\mathrm{V}$, respectively. The convergence of the VL-STCM-ID scheme is analysed in Section VI. In Section VII, the performance of the proposed schemes is discussed and finally our conclusions are offered in Section VIII.

\section{Space Time Coding Overview}

Let us consider a MIMO system employing $N_{t}$ transmit antennas and $N_{r}$ receive antennas. The signal to be transmitted from transmit antenna $m, 1 \leq m \leq N_{t}$, at the discrete time index $t$ is denoted as $x_{m}[t]$. The signal received at antenna $n$, $1 \leq n \leq N_{r}$, and at time instant $t$ can be modelled as:

$$
y_{n}[t]=\sqrt{E_{s}} \sum_{m=1}^{N_{t}} h_{n, m}[t] x_{m}[t]+w_{n}[t],
$$

where $E_{s}$ is the average energy of the signal constellation, $h_{n, m}[t]$ denotes the flat-fading channel coefficients between transmit antenna $m$ and receive antenna $n$ at time instant $t$, while $w_{n}[t]$ is the Additive White Gaussian Noise (AWGN) having zero mean and a variance of $N_{0} / 2$ per dimension. The amplitude of the modulation constellation points is scaled by a factor of $\sqrt{E_{s}}$, so that the average energy of the constellation points becomes unity and the expected Signal-to-Noise Ratio (SNR) per receive antenna is given by $\gamma=N_{t} E_{s} / N_{0}$ [11]. Let us denote the transmission frame length as $T$ symbol periods and define the space-time encoded codewords over $T$ symbol periods as an $\left(N_{t} \times T\right)$-dimensional matrix $\mathbf{C}$ formed as:

$$
\mathbf{C}=\left[\begin{array}{cccc}
c_{1}[1] & c_{1}[2] & \ldots & c_{1}[T] \\
c_{2}[1] & c_{2}[2] & \ldots & c_{2}[T] \\
\vdots & \vdots & \ddots & \vdots \\
c_{N_{t}}[1] & c_{N_{t}}[2] & \ldots & c_{N_{t}}[T]
\end{array}\right]
$$

where the elements of the $t$ th column $\mathbf{c}[\mathbf{t}]=$

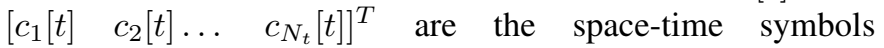
transmitted at time instant $t$ and the elements in the $m$ th row $\mathbf{c}_{\mathbf{m}}=\left[c_{m}[1] c_{m}[2] \ldots c_{m}[T]\right]$ are the space-time symbols transmitted from antenna $m$. The signal transmitted at time instant $t$ from antenna $m$, which is denoted as $x_{m}[t]$ in Equation 1, is the modulated space-time symbol given by $x_{m}[t]=f\left(c_{m}[t]\right)$ where $f($.$) is the modulator's$ mapping function. The Pair-Wise Error Probability (PWEP) of erroneously detecting $\mathbf{E}$ instead of $\mathbf{C}$ is upper bounded at high SNRs by [5], [12]:

$$
p(\mathbf{C} \rightarrow \mathbf{E}) \leq \frac{1}{2}\left(\frac{E_{s}}{4 N_{0}}\right)^{-E_{H} \cdot N_{r}}\left(E_{P}\right)^{-N_{r}},
$$

where $E_{H}$ is referred to as the effective Hamming distance, which quantifies the transmitter-diversity order and $E_{P}$ is termed as the effective product distance [5], which quantifies the coding advantage of a space-time code.

It was shown in [13], [14] that a full-spatial-diversity STTC scheme having the minimum decoding complexity can be systematically designed based on two steps. The first step is to design a block code, while the second step is to transmit the block code diagonally across the space-time grid. The mechanism of the diagonal transmission across the spacetime grid will be exemplified in Section IV in the context of Fig. 2. The Hamming distance and the product distance of a block code can be preserved, when the block code is transmitted diagonally across the space-time grid. Hence, a full-spatial-diversity STTC scheme can be realised, when the Hamming distance of the block code used by the STTC scheme equals to the number of transmitters. Based on the same principle, a joint source coding and STTC scheme can be systematically constructed by first designing a 2D VLC and then transmitting the 2D VLC diagonally across the space-time grid. As mentioned above [14], this allows us to achieve a transmitter-diversity order ${ }^{2}$, which is identical to the Hamming distance of the 2D VLC plus a coding advantage quantified by the product distance of the 2D VLC, as well as a multiplexing gain, provided that the number of possible source symbols $N_{s}$ is higher than the number of modulation levels $M$. More specifically, the spatial multiplexing gain is quantified by $\log _{2}\left(N_{s} / M\right)$ and the effective information rate of the scheme is given by $\eta=\log _{2}\left(N_{s}\right)$ bit/s/Hz.

Let us now commence our detailed discourse on the proposed VL-STCM-ID scheme in the following sections.

\section{TWO-DimENSIONAL VLC DESIGN}

Consider for example a source having $N_{s}=8$ possible discrete values and let the $l$ th value be represented by a symbol $s^{l}=l$ for $l \in\left\{1,2, \ldots, N_{s}\right\}$. Let us consider a source, where the symbols emitted are independent of each other, but the symbol probability distribution is not uniform and is given by:

$$
P\left(s^{l+1}\right)=0.6 P\left(s^{l}\right)=0.6^{l} P\left(s^{1}\right),
$$

and $\sum_{l=1}^{N_{s}} P\left(s^{l}\right)=1$. Hence, the source symbol $s^{1}$ has the highest occurrence probability of $P\left(s^{1}\right)=0.4068$ and the source symbol $s^{8}$ has the lowest occurrence probability of $P\left(s^{8}\right)=0.0114$. Note that a source is correlated, when its entropy rate $\mathcal{H}(s)$ is smaller than $\log _{2}\left(N_{s}\right)$ [15]. For the independent source considered, the source entropy rate equals the source entropy $H(s)$, which is given by: $\mathcal{H}(s)=H(s)=$

\footnotetext{
${ }^{2}$ The spatial-diversity order is the multiplication of the transmitter-diversity order and the receiver-diversity order.
} 


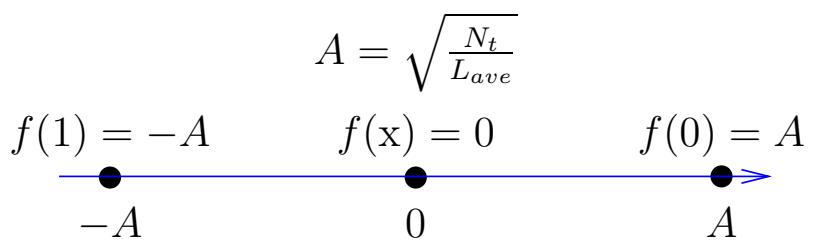

Fig. 1. The signal mapper of the VL-STCM.

$-\sum_{l=1}^{8} \log _{2}\left(P\left(s^{l}\right)\right) \cdot P\left(s^{l}\right)=2.302$ bit. Since $\mathcal{H}(s)<$ $\log _{2}\left(N_{s}\right)$, the source considered is a correlated source, where the higher the source correlation the smaller the source entropy rate. Let us now consider a 2D VLC codeword matrix, $V_{V L C}$, which encodes these $N_{s}=8$ possible source symbols using $N_{t}=3$ transmit antennas and BPSK modulation as follows:

$$
\mathbf{V}_{V L C}=\left[\begin{array}{llllllll}
\mathrm{x} & 1 & \mathrm{x} & 0 & \mathrm{x} & 0 & 1 & 1 \\
\mathrm{x} & \mathrm{x} & 0 & \mathrm{x} & 1 & 1 & 0 & 1 \\
0 & \mathrm{x} & \mathrm{x} & 1 & 1 & \mathrm{x} & 1 & 0
\end{array}\right] \text {, }
$$

where each column of the $(3 \times 8)$-dimensional matrix $\mathbf{V}_{V L C}$ corresponds to the specific VLC codeword conveying a particular source symbol and the elements in the matrix denoted as 0 and 1 represent the BPSK symbols to be transmitted by the $N_{t}=3$ transmit antennas, while ' $\mathrm{x}$ ' represents 'no transmission'. 'No transmission' implies that the corresponding transmit antenna sends no signal. Let the $l$ th source symbol $s^{l}$ be encoded using the $l$ th column of the $\mathbf{V}_{V L C}$ matrix seen in Equation 5. Hence, the source symbol $s^{1}$ is encoded into an $N_{t}$-element codeword using the first column of $\mathbf{V}_{V L C}$ in Equation 5, namely $\left[\begin{array}{lll}\mathrm{x} & \mathrm{x} & 0\end{array}\right]^{T}$, where the first and second transmit antennas are in the 'no transmission' mode, while the third antenna transmits an 'active' symbol represented by the binary value ' 0 '. If $L\left(s^{l}\right)$ is the number of 'active' symbols in the VLC codeword assigned to source symbol $s^{l}$, then we may define the average codeword length of the $2 \mathrm{D}$ VLC as:

$$
L_{\text {ave }}=\sum_{l=1}^{N_{s}} P\left(s^{l}\right) L\left(s^{l}\right),
$$

where we have $L_{\text {ave }}=1.233 \mathrm{bit} / \mathrm{VLC}$ codeword for this system according to Equations 4 and 5 .

The corresponding BPSK signal mapper is characterised in Fig. 1, where the 'no transmission' symbol is actually represented by the origin of the Euclidean space, i.e. we have $f(\mathrm{x})=0$, where $f($.$) is the mapping function. Since the "no$ transmission' symbol is a zero energy symbol, the amount of energy saving can be computed from:

$$
A^{2}=\frac{N_{t}}{L_{\text {ave }}}
$$

where we have $A^{2}=3 / 1.233=2.433$, which is equivalent to $20 \log (A)=3.86 \mathrm{~dB}$. Hence, more transmitted energy is saved, when there are more 'no transmission' symbols in a VLC codeword. Therefore, the columns of the matrix $\mathbf{V}_{V L C}$ in Equation 5, which are the VLC codewords, and the source symbols are specifically arranged, so that the more frequently occurring source symbols are assigned to VLC codewords having more 'no transmission' components, in order to save transmit energy. The energy saved is then reallocated to the 'active' symbols for the sake of increasing their minimum Euclidean distance, as shown in Fig. 1.

Let us define the Hamming distance $E_{H \text { min }}$ as the number of different symbol positions of all the columns in the 2D VLC codeword matrix. Hence, we have $E_{H \text { min }}=2$ for the 2D VLC codeword matrix in Equation 5. We further define the minimum product distance $E_{P \text { min }}$ as:

$$
E_{P \min }=\min _{1 \leq s<\tilde{s} \leq N_{s}} \prod_{m \in \xi}\left|f\left(v_{m}\right)-f\left(\tilde{v}_{m}\right)\right|^{2},
$$

where $\xi$ represents the set of VLC codeword component indices $m$ satisfying the condition of $v_{m} \neq \tilde{v}_{m}$ for $1 \leq m \leq$ $N_{t}$ and the VLC codeword $\mathbf{v}=\left[\begin{array}{llll}v_{1} & v_{2} & \ldots & v_{N_{t}}\end{array}\right]^{T}$ is defined in Fig. 2 and Equation 5. The two VLC codewords conveying the source symbols $s$ and $\tilde{s}$ are represented as $\left[v_{1} \ldots v_{N_{t}}\right]^{T}$ and $\left[\tilde{v}_{1} \ldots \tilde{v}_{N_{t}}\right]^{T}$, respectively, in Equation 8. We have $E_{P \text { min }}=$ 5.92 based on Equation 5 and the signal mapper of Fig. 1. Note that the mapper function $f($.$) used in Equation 8$ and portrayed in Fig. 1 depends on the amount of energy saving. The amount of energy saving is given by Equation 7, where the numerator $N_{t}$ is fixed and the denominator $L_{a v e}$ is given by Equation 6. As in the conventional 1D VLC, the higher the source correlation, the lower the average codeword length $L_{\text {ave }}$ of the 2D VLC. A higher energy saving can be attained, when the source is more correlated due to a reduced average codeword length $L_{\text {ave }}$. In other words, the source correlation is converted into an increased minimum product distance, resulting in an increased coding gain, when the source is more correlated. By contrast, the conventional 1D VLC exploits the source correlation for attaining an increased compression ratio.

The design of the 2D VLC scheme can be summarised in the following three steps:

1) Search for all possible VLC codeword matrices, which have the maximum achievable minimum Hamming distance $E_{H \text { min }}$ and product distance $E_{P \text { min }}$ values for each pair of the VLC codewords at a given $N_{s}$ and $N_{t}$ combination. Note that attaining a higher $E_{H \text { min }}$ is given more weight than $E_{P \text { min }}$, since $E_{H \text { min }}$ is more dominant in the PWEP of Equation 3.

2) Rearrange the columns of the VLC codeword matrices in descending orders according to the number of 'no transmission' components. Assign the source symbols to the columns of the VLC codeword matrix, in descending orders according to the symbol probabilities.

3) Find the VLC codeword matrix that gives the shortest average codeword length with the aid of Equation 6.

Note that the search-space of step 1 can be significantly reduced with the aid of the branch-and-bound algorithm of [16], where both $E_{H \text { min }}$ and $E_{P \text { min }}$ are used during the bounding operation. By contrast, the code search in [8], [14] also employs the branch-and-bound algorithm, but uses only $E_{P \text { min }}$ in the bounding operation. The 2D VLC matrix seen in Equation 5 was designed based on the above three steps. When $N_{t}=3$ transmit antennas are employed for transmitting the $N_{t}$-element 2D VLC codewords denoted as $\mathbf{v}=\left[v_{1} \ldots v_{N_{t}}\right]^{T}$ in Fig. 2, we achieve a transmitter-diversity order of $E_{H \text { min }}=$ 2, a coding gain quantified by $E_{P \min }=5.92$ and a spatial multiplexing gain quantified by $\log _{2}\left(N_{s} / M\right)=2$, where $M=$ 2 is the number of modulation levels of the original BPSK 


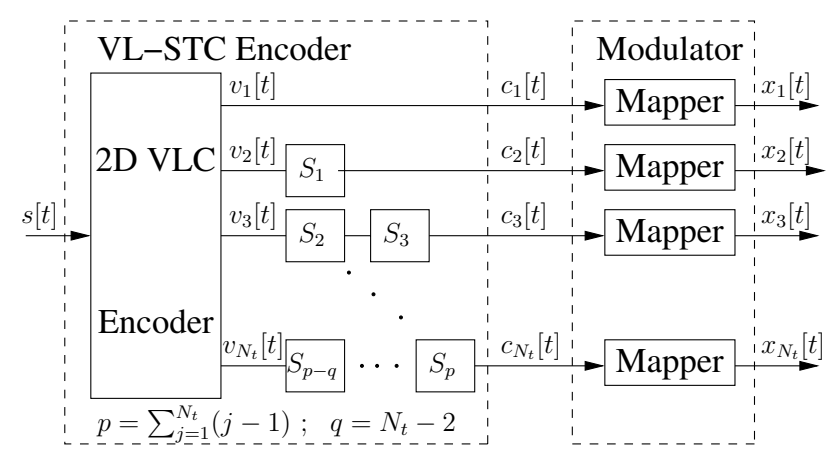

Fig. 2. Block diagram of the VL-STCM transmitter.

modulation and $N_{s}=8$ is the number of source symbols. The throughput of the scheme is given by $\eta=\log _{2}\left(N_{s}\right)=3$ $\mathrm{bit} / \mathrm{s} / \mathrm{Hz}$ and the Signal to Noise Ratio (SNR) per bit is given by $E_{b} / N_{0}=\gamma / \eta$, where $\gamma$ is the SNR per receive antenna.

Based on the above design principles, a range of 2D VLCs can be created for $M$-ary PSK modulation for $M>2$, where the origin of the Euclidean space represents the "no transmission' symbol. In general, a VLC code that has the lowest average codeword length $L_{a v e}$ is attractive in terms of energy saving (or source compression for 1D VLC). However, we found that decoding convergence ${ }^{3}$ could not be achieved when the minimum Hamming distance $E_{H \text { min }}$ of the 1D/2D VLCs equals unity. Hence the tradeoff between energy saving (or source compression for 1D VLC) and decoding gain lies between minimising $L_{a v e}$ and maximizing $E_{H \text { min }}$. Our goal is to design a 2D VLC which could help to provide an overall performance approaching the channel capacity, when it is employed in the iteratively detected MIMO system.

\section{VL-STCM SCHEME}

The block diagram of the VL-STCM transmitter is illustrated in Fig. 2, which can be represented by two fundamental blocks, namely the Variable Length Space Time Code (VLSTC) encoder and the modulator. As seen in Fig. 2, a VLC

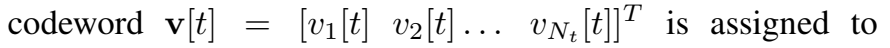
each of the source symbols $s[t]$ generated by the source at time instant $t$, where we have $s[t] \in\left\{1, \ldots, N_{s}\right\}$ and $N_{s}$ denotes the number of possible source symbols. Each of the VLC codewords $\mathbf{v}[t]$ seen in Fig. 2 corresponds to one of the columns in the VLC matrix of Equation 5. Hence, the component $v_{m}[t]$ of the VLC codeword is represented by a symbol seen in the VLC matrix of Equation 5. As portrayed in Fig. 2, the VLC codeword $\mathbf{v}[t]$ is transmitted diagonally across the space-time grid with the aid of appropriate-length shift registers denoted as $S_{k}$ in Fig. 2, where we have $k \in$ $\left\{1,2, \ldots, \sum_{j=1}^{N_{t}}(j-1)\right\}$. As we can see from Fig. 2, the

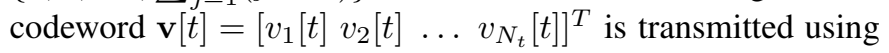
$N_{t}$ transmit antennas, where the $m$ th element of each VLC codeword, for $1 \leq m \leq N_{t}$, is delayed by $(m-1)$ shift register cells, before it is transmitted through the $m$ th transmit antenna. Hence, the $N_{t}$ number of components of each VLC codeword are transmitted on a diagonal of the space-time codeword matrix of Equation 2. Since the VLC codewords

\footnotetext{
${ }^{3}$ The convergence analysis of the system will be detailed in Section VI.
}

TABLE I

The Space-Time Codeword TABle

\begin{tabular}{|c|c|c|c|c|c|}
\hline Index & c & Index & c & Index & c \\
\hline 0 & {$\left[\begin{array}{llll}0 & \mathrm{x} & \mathrm{x}\end{array}\right]^{T}$} & 9 & {$\left[\begin{array}{llll}0 & 0 & \mathrm{x}\end{array}\right]^{T}$} & 18 & $\begin{array}{llll}{\left[\begin{array}{lll}0 & 1 & \mathrm{x}\end{array}\right]^{T}} \\
\end{array}$ \\
\hline 1 & {$\left[\begin{array}{llll}0 & \mathrm{x} & 1\end{array}\right]^{T}$} & 10 & {$\left[\begin{array}{llll}0 & 0 & 1\end{array}\right]^{T}$} & 19 & {$\left[\begin{array}{llll}0 & 1 & 1\end{array}\right]^{T}$} \\
\hline 2 & {$\left[\begin{array}{llll}0 & \mathrm{x} & 0\end{array}\right]^{T}$} & 11 & {$\left[\begin{array}{llll}0 & 0 & 0\end{array}\right]^{T}$} & 20 & {$\left[\begin{array}{llll}0 & 1 & 0\end{array}\right]^{T}$} \\
\hline 3 & {$\left[\begin{array}{llll}1 & \mathrm{x} & \mathrm{x}\end{array}\right]^{T}$} & 12 & {$\left[\begin{array}{llll}1 & 0 & \mathrm{x}\end{array}\right]^{T}$} & 21 & {$\left[\begin{array}{llll}1 & 1 & \mathrm{x}\end{array}\right]^{T}$} \\
\hline 4 & {$\left[\begin{array}{lll}1 & \mathrm{x} & 1\end{array}\right]^{T}$} & 13 & {$\left[\begin{array}{lll}1 & 0 & 1\end{array}\right]^{T}$} & 22 & {$\left[\begin{array}{llll}1 & 1 & 1\end{array}\right]^{T}$} \\
\hline 5 & {$\left[\begin{array}{llll}1 & \mathrm{x} & 0\end{array}\right]^{T}$} & 14 & {$\left[\begin{array}{llll}1 & 0 & 0\end{array}\right]^{T}$} & 23 & {$\left[\begin{array}{lll}1 & 1 & 0\end{array}\right]^{T}$} \\
\hline 6 & {$\left[\begin{array}{llll} & \mathrm{x} & \mathrm{x} & \mathrm{x}\end{array}\right]^{T}$} & 15 & {$\left[\begin{array}{llll} & \mathrm{x} & 0 & \mathrm{x}\end{array}\right]^{T}$} & 24 & {$\left[\begin{array}{lll}\mathrm{x} & 1 & \mathrm{x}\end{array}\right]^{T}$} \\
\hline 7 & {$\left[\begin{array}{lll}\mathrm{x} & \mathrm{x} & 1\end{array}\right]^{T}$} & 16 & {$\left[\begin{array}{llll}\mathrm{x} & 0 & 1\end{array}\right]^{T}$} & 25 & {$\left[\begin{array}{llll}\mathrm{x} & 1 & 1\end{array}\right]^{T}$} \\
\hline 8 & {$\left[\begin{array}{llll}\mathrm{x} & \mathrm{x} & 0\end{array}\right]^{T}$} & 17 & {$\left[\begin{array}{llll}\mathrm{x} & 0 & 0\end{array}\right]^{T}$} & 26 & {$\left[\begin{array}{llll}\mathrm{x} & 1 & 0\end{array}\right]^{T}$} \\
\hline
\end{tabular}

are encoded diagonally, the space-time coded symbol $c_{m}[t]$ transmitted by the $m$ th antenna, $1 \leq m \leq N_{t}$, at a particular time-instant $t$ is given by $c_{m}[t]=v_{m}[t-m+1]$. Hence, for this specific case the transmitted signal is given by:

$$
x_{m}[t]=f\left(c_{m}[t]\right)=f\left(v_{m}[t-m+1]\right),
$$

for $1 \leq m \leq N_{t}$. Note that originally there were only $N_{s}=8$ legitimate 2D-VLC codewords in Equation 5. However, after these VLC codewords are diagonally mapped across the spacetime grid using the shift registers shown in Fig. 2, there is a total of $\bar{M}^{N_{t}}=3^{3}=27$ legitimate space-time codewords ${ }^{4}$, where $\bar{M}$ is the number of possible symbols in each position of the 2D VLC codewords. Note however that the number of legitimate space-time codewords may become lower than $\bar{M}^{N_{t}}$ when a different 2D VLC codeword matrix is employed.

The corresponding trellis diagram of the proposed VL-STC encoder is depicted in Fig. 3. The $N_{t}=3$-element space-time codeword seen in Fig. 2 is given by $\mathbf{c}=\left[\begin{array}{lll}c_{1} & c_{2} & c_{3}\end{array}\right]^{T}$. The relationship between the 27 codeword indices shown in Fig. 3 and the space-time codeword $\mathbf{c}$ is defined in Table I. The trellis states are defined by the contents of the shift register cells $S_{k}$ shown in Fig. 2, which are denoted by $\mathbf{S}=\left[\begin{array}{lll}S_{1} & S_{2} & S_{3}\end{array}\right]$. For example, the state $\mathbf{S}=0$ is denoted as $\left[\begin{array}{lll}x & 0 & 0\end{array}\right]$ in the trellis diagram of Fig. 3. Note that each shift register cell may hold $\bar{M}=3$ possible values, namely $\{0,1, \mathrm{x}\}$ in conjunction with the VL-STC encoder based on Equation 5. However, the number of legitimate trellis states can be less than $\bar{M}^{p}$, where $p=\sum_{j=1}^{N_{t}}(j-1)=3$ is the total number of shift registers. Hence, in our specific case there are only 24 legitimate trellis states out of the $\bar{M}^{p}=27$ possible trellis states, which is a consequence of the constraints imposed by the 2D VLC of Equation 5. As we can see from Fig. 3, there are always $N_{s}=8$ diverging trellis branches from each state due to the eight possible source symbols. However, the number of converging trellis paths may vary from one state to another due to the variable length structure of the space-time codewords. Explicitly, there are six and nine trellis paths converging to state $\mathbf{S}=0$ and state $\mathbf{S}=1$, respectively, as seen in the trellis structure of Fig. 3.

Similar to the full-spatial-diversity STTC scheme of [14], the above VL-STCM design has the minimum decoding complexity required for attaining the target transmitter-diversity and multiplexing gain. On one hand, it is possible to design a range of higher-complexity VL-STCM schemes in order to

\footnotetext{
${ }^{4} \mathrm{~A}$ space-time codeword is defined as the $N_{t}$-element output word of the VL-STC encoder of Fig. 2.
} 
$\mathbf{c}:\left[c_{1} c_{2} c_{3}\right]^{\mathrm{T}} \quad \mathbf{S}:\left[S_{1} S_{2} S_{3}\right]$

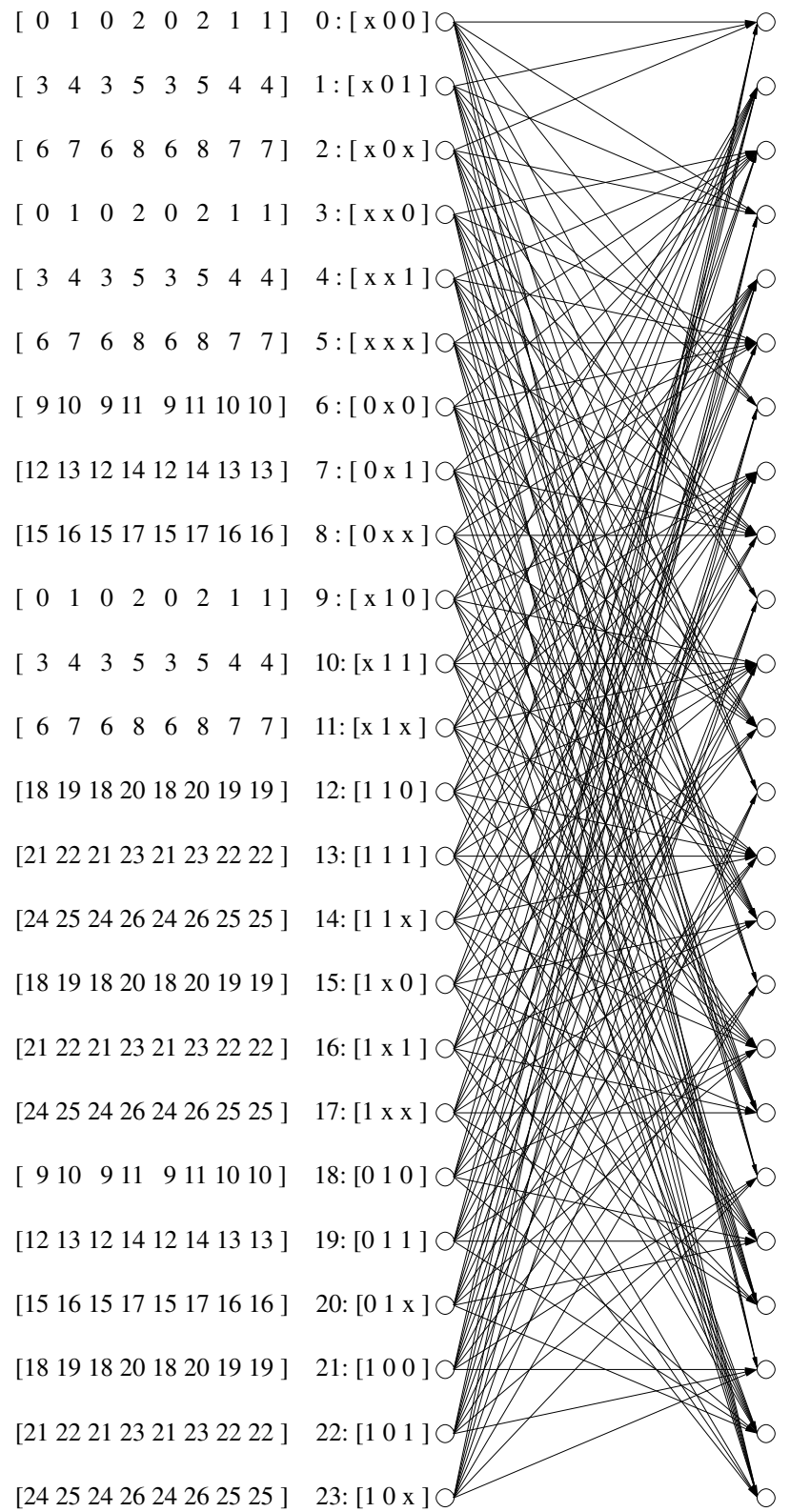

Fig. 3. The trellis of VL-STC encoder when invoking the 2D VLC of Equation 5. For the list of codewords see Table I.

attain a higher coding gain. On the other hand, iterative decoding is well known for achieving near-channel-capacity performance with the aid of low complexity constituent codes [17]. Hence, we will employ this minimum-complexity transmitterdiversity and multiplexing based VL-STCM arrangment as one of the constituent codes in an iterative decoding scheme.

\section{VL-STCM-ID SCHEME}

In order to invoke iterative detection and hence attain iteration gains as a benefit of the more meritoriously spread extrinsic information, we introduce a symbol-based random ${ }^{5}$ interleaver and a non-binary URC for each of the $N_{t}=$ 3 transmit antennas. The $N_{t}=3$ parallel symbol-based

\footnotetext{
${ }^{5}$ The optimumisation of the interleaver is not considered in this paper.
}

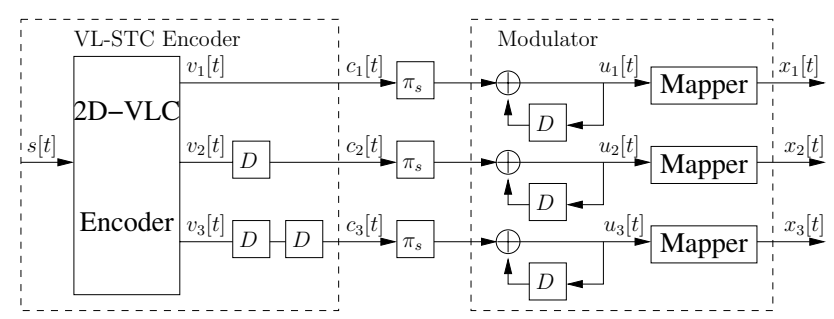

Fig. 4. The VL-STCM-ID transmitter employing $N_{t}=3$ transmit and $N_{r}=2$ receive antennas, where $\pi_{s}$ denotes symbol interleaver.

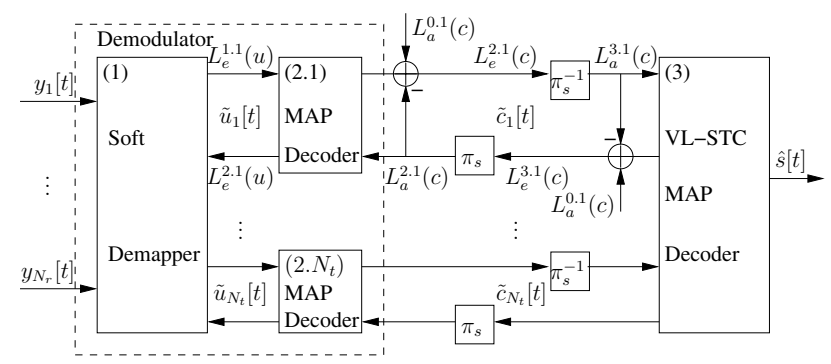

Fig. 5. The VL-STCM-ID receiver for an $N_{r} \times N_{t}$ MIMO system. The notation $(\tilde{.})$ and $(\hat{.})$ indicates the extrinsicla priori probability and the hard decision estimate of (.), respectively. The notation $L_{(a, e)}^{i . m}(c, u)$ denotes the log-domain symbol probability of the VL-STC codeword $c$ or the URC codeword $u$ for the $m$ th transmitter. The subscripts $a$ and $e$ denote the a priori and extrinsic nature of the probabilities while the superscript $i . m$ identifies that the probabilities belong to the $i$ th stage decoder for the $m$ th transmitter. Note that $i=0$ means that the probabilities were calculated from the source symbol distribution.

interleavers were generated independently. As we can see from Fig. 4, each element in the space-time codeword $c_{m}[t]$ for $m \in\{1,2,3\}$ is further interleaved and encoded by a non-binary URC, before feeding them to the mapper. The convergence behaviour of the iterative VL-STCM-ID decoder will be analysed in Section VI, where it will be shown that the VL-STCM-ID scheme would be unable to converge at low SNRs, when the recursive feedback assisted non-binary precoders or URCs are not used. By contrast, a simple singlecell non-binary URC invoked before the mapper of each transmit antenna would allow us to achieve a significant iteration gain. Hence, the VL-STC encoder was retained unaltered, but the original modulator was modified. The non-binary URC employs a modulo- $\bar{M}$ adder, where again, $\bar{M}=3$ is the number of different symbols in the VLC space-time codeword and we have $c_{m}[t] \in\{0,1, \mathbf{x}\}$. Accordingly, this single-cell URC possesses $\bar{M}=3$ trellis states. Note that we represent the ' $\mathrm{x}$ ' symbol using the number ' 2 ' during the modulo$\bar{M}$ addition. Furthermore, the VL-STCM-ID scheme is very similar to the BICM-ID arrangement proposed in [18], where $N$ parallel bit-based interleavers were used for interleaving the bits of the $N$-bit codeword before the modulator, in order to attain iteration gains. By contrast, the VL-STCM-ID scheme employs $N_{t}$ parallel symbol-based interleavers for interleaving the symbols of the $N_{t}$-symbol space-time codeword before the modulator, in order to attain iteration gains with the aid of iterative decoding.

At the receiver, the symbol-based log-domain MAP algorithm [17] is used by both the VL-STC decoder and the URC decoder. The block diagram of the VL-STCM-ID receiver is 
depicted in Fig. 5, where we denote the log-domain symbol probability of the VL-STC codeword $c_{m}$ and the URC codeword $u_{m}$ for the $m$ th transmitter as $L_{(a, e)}^{i . m}(c)$ and $L_{(a, e)}^{i . m}(u)$, respectively. Furthermore, the subscripts $a$ and $e$ denote the a priori and extrinsic nature of the probabilities, while the superscript $i . m$ suggests that the probabilities belong to the $i$ th decoder stage of the $m$ th transmitter. Note that $i=0$ implies that the probabilities were calculated from the source symbol distribution. The extrinsic probability of the URC codeword of transmit antenna $m$, namely $P_{e}\left(u_{m}[t]\right)$, can be computed during each symbol period in the 'Soft Demapper' block of Fig. 5. By dropping the time-related square bracket, we can compute $P_{e}\left(u_{m}\right)$ as:

$$
\begin{array}{r}
P_{e}\left(u_{m}=b\right)=\sum_{\substack{\mathbf{x} \in \chi(m, b) \\
m \in\left\{1,2, \ldots, N_{t}\right\}, b \in\{0,1, \mathbf{x}\}}}\left(P(\mathbf{y} \mid \mathbf{x}) \prod_{j \neq m}^{\text {all } j} P_{a}\left(u_{j}\right)\right),
\end{array}
$$

where the subset $\chi(m, b)$ contains all the phasor combinations for the transmitted signal vector $\mathbf{x}=\left[\begin{array}{llll}x_{1} & x_{2} & \ldots & x_{N_{t}}\end{array}\right]^{T}$ where $x_{m}=f\left(u_{m}=b\right)$ holds, while $P(\mathbf{y} \mid \mathbf{x})$ is the Probability Density Function (PDF) of the MIMO Rayleigh fading channel given by:

$$
P(\mathbf{y} \mid \mathbf{x})=\frac{1}{\left(2 \pi \sigma_{n}^{2}\right)^{N_{r}}} \exp \left(\frac{-\|\mathbf{y}-\mathbf{H} \mathbf{x}\|^{2}}{2 \sigma_{n}^{2}}\right),
$$

and $\sigma_{n}^{2}=N_{0} / 2$ is the noise variance, $\mathbf{y}$ is the $N_{r}$-element complex received signal vector, while $\mathbf{H}$ is an $\left(N_{r} \times N_{t}\right)$ dimensional complex channel matrix during the time instant $t$. Furthermore, the a priori probability of $u_{m}$ in Equation 10 is computed from the extrinsic log-domain probability of the $m$ th URC MAP decoder as $P_{a}\left(u_{m}\right)=\exp \left(L_{e}^{2 . m}(u)\right)$, while the log-domain a priori probability of $u_{m}$ for the $m$ th URC MAP decoder is given by $L_{e}^{1 . m}(u)=\ln \left(P_{e}\left(u_{m}\right)\right)$. Hence, the soft demapper benefits from the a priori information of its input symbols $u_{m}$ after the first iteration. Note that we employ the Jacobian logarithm [19] to compute Equation 10 in the log-domain, hence there is no need for log-domain to normal-domain conversion. As we can see from Fig. 5, each of the $N_{t}=3$ URC MAP decoders seen inside the demodulator block benefits from the a priori information of its codeword $u_{m}[t]$ as well as from that of its input symbol $c_{m}[t], m \in$ $\left\{1,2, \ldots, N_{t}\right\}$.

It is possible to attain some a priori probability for the $N_{t}$-element VL-STC codewords, c, (which also constitute the URC's input words), given the source symbol occurrence probability specified in Equation 4. Explicitly, the probability of the $m$ th URC's input word $c_{m}$ can be expressed as:

$$
\begin{aligned}
P\left(c_{m}=d\right)= & \sum_{\substack{l \in \mu(m, d)\\
}} P\left(s^{l}\right), \\
& m \in\left\{1,2, \ldots, N_{t}\right\}, d \in\{0,1, \mathrm{x}\},
\end{aligned}
$$

where the subset $\mu(m, d)$ contains the specific indices of those columns in the VLC matrix, where the $m$ th row element in that column equals $d$. Hence, we have $L_{a}^{0 . m}(c)=\ln \left(P\left(c_{m}\right)\right)$ as an additional a priori probability for symbol $c_{m}$ during each iteration between the URC MAP decoder and the VLSTC MAP decoder, as shown in Fig. 5. Note that $P\left(c_{m}\right)$ is directly computed from the source symbol occurrence probability $P\left(s^{l}\right)$, hence we do not use $P\left(s^{l}\right)$ again as the a priori probability of the VL-STC input word in the VL-STC MAP decoder, in order to avoid reusing the same information. Therefore, both the VL-STC and the URC MAP decoders benefit from the a priori or extrinsic information of the space-time codeword $\mathbf{c}[t]=\left[c_{1}[t] c_{2}[t] c_{3}[t]\right]^{T}$ received from each other as well as from the additional a priori information provided by the potentially different probability source symbols $s[t]$. A full iteration consists of a soft demapper operation, $N_{t}=3$ URC MAP decoder operations and a VL-STC MAP decoder operation. For the non-iteratively decoded VLSTCM/FL-STCM, the soft demapper computes the a priori

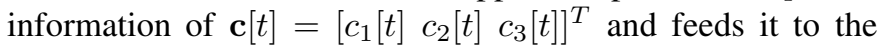
VL-STC/FL-STCM MAP decoder. Note that the VL-STC/FLSTC decoder of VL-STCM/FL-STCM also benefits from the a priori probability of its input word $s[t]$, which is given by the source symbol occurrence probability in Equation 4 . Hence, as the source becomes correlated, the VL-STCMID, VL-STCM and FL-STCM schemes will benefit from the a priori probability of the source symbols. However, FLSTCM attains no energy savings.

\section{Convergence Analysis}

Extrinsic Information Transfer (EXIT) charts designed for binary receivers [20] have been widely used for analysing the convergence behaviour of iterative decoding aided concatenated coding schemes. The non-binary EXIT charts were introduced on the basis of the multi-dimensional histogram computation of [21], [22]. However, the convergence analysis of the proposed three-stage VL-STCM-ID scheme requires the employment of novel three Dimensional (3D) non-binary EXIT charts, which evolved from the binary 3D EXIT charts used in [23], [24] for analysing multiple concatenated codes.

To elaborate a little further, EXIT charts visualise the input and output characteristics of the constituent MAP decoders in terms of the mutual information transfer between the input sequence and the a priori information at the input, as well as between the input sequence and the extrinsic information at the output of the constituent decoder. Hence, there are two steps in generating an EXIT chart. Firstly, we have to model the $a$ priori probabilities of the input sequence and then feed it to the decoder. Secondly, we have to compute the mutual information of the extrinsic probabilities at the output of the decoder. Let us now model the a priori probabilities of the VL-STC codeword, $\mathbf{c}=\left[\begin{array}{llll}c_{1} & c_{2} & \ldots & c_{N_{t}}\end{array}\right]^{T}$, where $c_{m}$, $m \in\left\{1,2, \ldots, N_{t}\right\}$, is also the input symbol of the $m$ th URC.

Let us denote the input symbol of the $m$ th URC as $c$, where the subscript $m$ is omitted for simplicity. Assume that the symbol $c$ is transmitted across an AWGN channel using the $\mathcal{M}=3$-phasor mapper shown in Fig. 1 and the received signal is given by $y=x+n$, where $n$ is the AWGN noise having a zero mean and a variance of $\bar{\sigma}_{n}^{2}$. Furthermore, we have $x=f(c)$, where $f($.$) is the mapper function portrayed$ in Fig. 1. Since $f($.$) is a memoryless function, the probability$ of occurrence for $x$ is the same as that of $c$. Hence, we have $P(x)=P(c)$, which can be expressed from Equation 12. At a given probability of occurrence for $x$, the mutual information 
between $x$ and $y$ can be formulated as:

$$
\begin{aligned}
I(x, y) & =\sum_{i=1}^{\mathcal{M}} \int_{y} P\left(x_{i}, y\right) \log _{2}\left(\frac{P\left(x_{i}, y\right)}{P\left(x_{i}\right) P(y)}\right) d y \\
& =H(x)-H(x \mid y)
\end{aligned}
$$

where $H(x)$ is the entropy of $x$, given by:

$$
H(x)=-\sum_{i=1}^{\mathcal{M}} P\left(x_{i}\right) \log _{2}\left(P\left(x_{i}\right)\right),
$$

and $H(x \mid y)$ is the conditional entropy of $x$ given $y$, which can be expressed as:

$$
H(x \mid y)=\sum_{i=1}^{\mathcal{M}} P\left(x_{i}\right) E\left[\log _{2}\left(\sum_{j=1}^{\mathcal{M}} \frac{P\left(x_{j}\right)}{P\left(x_{i}\right)} \exp \left(\Psi_{i, j}\right)\right)\right],
$$

where $\exp \left(\Psi_{i, j}\right)=P\left(y \mid x_{j}\right) / P\left(y \mid x_{i}\right)$ and $P(y \mid x)$ is the conditional Gaussian PDF, while the exponent $\Psi_{i, j}$ is given by:

$$
\Psi_{i, j}=\frac{-\left|x_{i}-x_{j}+n\right|^{2}+|n|^{2}}{2 \bar{\sigma}_{n}^{2}} .
$$

The expectation term $E[$.$] in Equation 15$ is taken over different representations of the AWGN noise $n$. Hence, a curve can be generated for $I(x, y)$ versus $\bar{\sigma}_{n}^{2}$, where the expectation term in Equation 15 is evaluated using Monte Carlo simulation. We can simplify Equation 13 to a form, where $I(x, y)$ is expressed as a function of $\bar{\sigma}_{n}^{2}$. Let us denote this function as $J($.$) and we have I(x, y)=J\left(\bar{\sigma}_{n}^{2}\right)$. Note that $I(x, y)$ is monotonically decreasing with respect to $\bar{\sigma}_{n}^{2}$.

Let us now denote the a priori information of $c$ as $I_{A}(c)=$ $I(x, y)$. At a given $I_{A}$ value we can find the corresponding noise variance with the aid of the inverse function $\bar{\sigma}_{n}^{2}=$ $J^{-1}\left(I_{A}(c)\right)$ using the $I(x, y)$ versus $\bar{\sigma}_{n}^{2}$ curve. Then we can generate a noise sample $n^{\prime}$ having a variance of $\bar{\sigma}_{n}^{2}$. Consequently, we can produce $y^{\prime}=x+n^{\prime}$, where again $x=f(c)$ represents the mapper function portrayed in Fig. 1 and $c$ is the actual input symbol of the $m$ th URC. Finally, we can generate the a priori symbol probabilities for $P_{a}(c)$ using the conditional Gaussian PDF:

$$
P_{a}(c)=\frac{1}{2 \pi \bar{\sigma}_{n}^{2}} \exp \left(\frac{-\left|y^{\prime}-f(c)\right|^{2}}{2 \bar{\sigma}_{n}^{2}}\right),
$$

for $c \in\{0,1, \mathrm{x}\}$. Then we feed these symbol probabilities to the corresponding MAP decoder. Note that the above method can be used for any symbol-interleaved serially concatenated coding schemes, where the symbol probabilities are directly created for a given $I_{A}$ value. The mutual information for the $N_{t}$-element VL-STC codeword $\mathbf{c}=\left[\begin{array}{llll}c_{1} & c_{2} & \ldots & c_{N_{t}}\end{array}\right]^{T}$ is the sum of the mutual information valid for its symbol components $c_{m}$, expressed as $I_{A}(\mathbf{c})=\sum_{m=1}^{N_{t}} I_{A}\left(c_{m}\right)$, where $I_{A}\left(c_{m}\right)$ is the mutual information of the $m$ th symbol component of the VL-STC codeword or the $m$ th URC's input symbol, given by Equation 13. Note that the maximum $I_{A}\left(c_{m}\right)$ value equals the entropy of $c_{m}$ given by Equation 14 .

Next, we compute the mutual information of the extrinsic symbol probabilities $I_{E}\left(c_{m}\right)$ at the output of the VL-STC or URC decoder for the symbol $c_{m}$ using the method proposed in[25], which is computationally more efficient compared

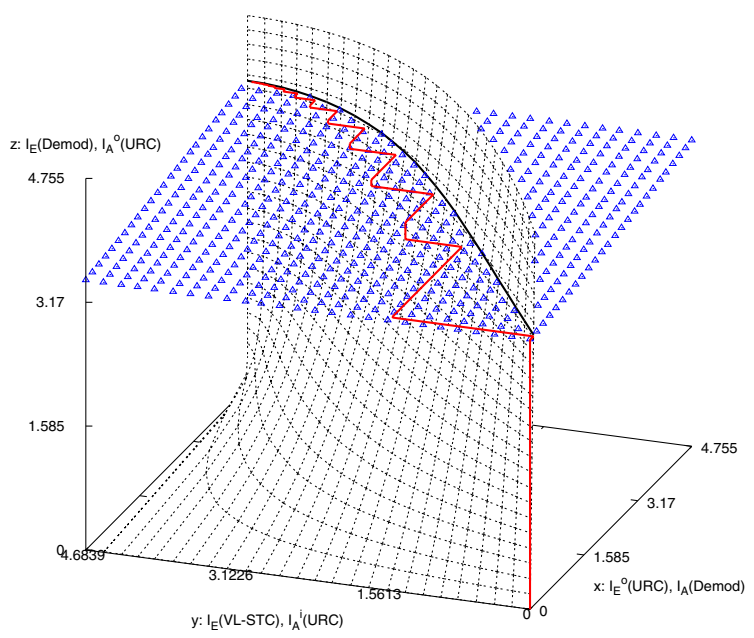

Fig. 6. The 3D EXIT charts for the VL-STCM-ID scheme having $N_{t}=3$ and $N_{r}=2$, when using an uncorrelated source. The iterative trajectory is computed at $E_{b} / N_{0}=4 \mathrm{~dB}$. The maximum value of an axis denotes the entropy of the corresponding symbol.

to [21]. Finally, the mutual information of the extrinsic symbol probabilities for the VL-STC codeword can be computed from $I_{E}(\mathbf{c})=\sum_{m=1}^{N_{t}} I_{E}\left(c_{m}\right)$. We also compute the mutual information for the URC codeword $\mathbf{u}$ based on the same procedure. However, the experimentally measured PDF histogram of $\mathbf{u}$ was found to be near-uniform associated with equiprobable symbol, when the symbol interleaver length was sufficiently high. This is because the URC employed can be viewed as an accumulator.

The 3D EXIT charts and the actual iterative decoding trajectories for the VL-STCM-ID scheme having $N_{t}=3$ and $N_{r}=2$ when using an uncorrelated source are shown in Figs. 6 and 7, respectively. Let us denote the three axes of the 3D EXIT charts using the letters $\mathrm{x}, \mathrm{y}$ and $\mathrm{z}$, while $I_{A}(*)$ and $I_{E}(*)$ denote the a priori and extrinsic information for $(*)$, respectively, where $\left(^{*}\right)$ is either the VL-STC MAP decoder (VL-STC) or the URC MAP decoder (URC) or, alternatively, the soft demapper (Demod). As we can see from Fig. 5, each of the URC MAP decoders takes (provides) the a priori (extrinsic) probabilities of its input word $c$ and output word (or codeword) $u$ as the input (output). Hence, the mutual information of the input word and output word of the URC decoder will be represented by $I_{(A, E)}^{i}$ (URC) and $I_{(A, E)}^{o}(\mathrm{URC})$, respectively. Each of the $N_{t}$ symbol interleavers shown in Figs. 4 has a length of 10000 symbols.

The EXIT plane marked with triangles in Fig. 6 was computed based on the extrinsic probabilities of the soft demapper $L_{e}^{1 . m}(u)$, for $m \in\{1,2,3\}$, at the given values in the $\mathrm{x}$ and $\mathrm{y}$ axes at $E_{b} / N_{0}=4 \mathrm{~dB}$. The other EXIT plane marked with dashed lines in Fig. 6 is SNR-independent and was plotted based on the URC decoders' output word extrinsic probabilities $L_{e}^{2 . m}(u)$, for $m \in\{1,2,3\}$, at the given values in the $\mathrm{y}$ and $\mathrm{z}$ axes. The decoding trajectory, which was determined based on the extrinsic probabilities 


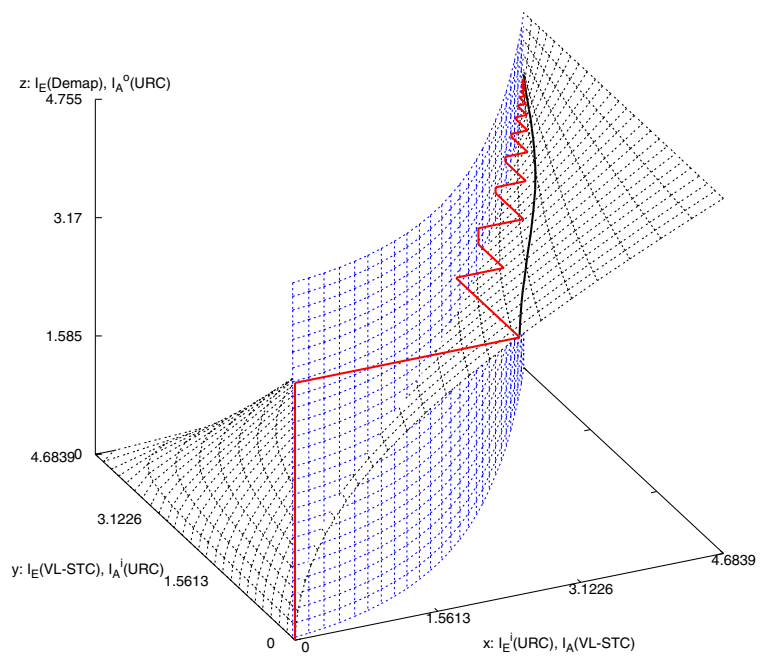

Fig. 7. The 3D EXIT charts for the VL-STCM-ID scheme having $N_{t}=3$ and $N_{r}=2$, when using an uncorrelated source. The iterative trajectory is computed at $E_{b} / N_{0}=4 \mathrm{~dB}$. The maximum value of an axis denotes the entropy of the corresponding symbol.

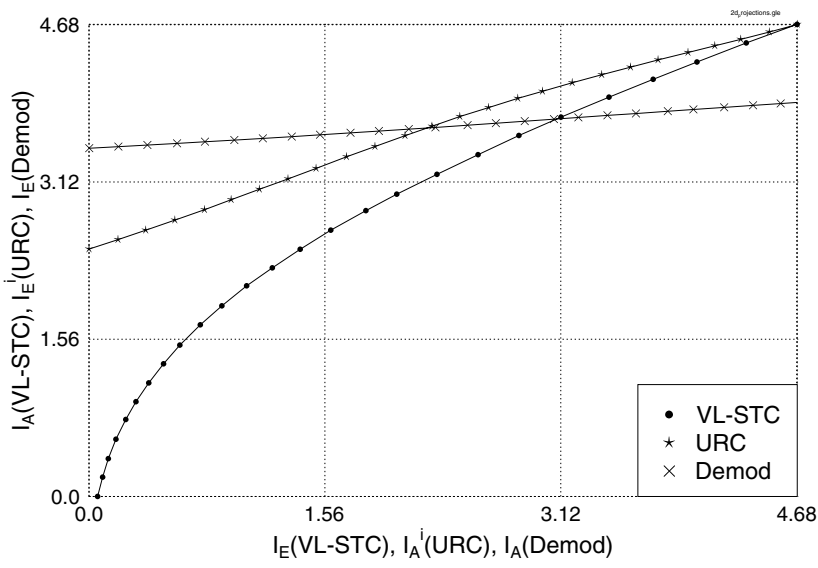

Fig. 8. The 2D EXIT charts projection for the VL-STCM-ID scheme having $N_{t}=3$ and $N_{r}=2$, when using an uncorrelated source at $E_{b} / N_{0}=4 \mathrm{~dB}$. The maximum value of an axis denotes the entropy of the corresponding symbol.

$L_{e}^{1 . m}(\mathrm{u}), L_{e}^{2 \cdot m}(u)$ and $L_{e}^{3 \cdot m}(c)$ for $m \in\{1,2,3\}$ as in Fig. 5, is under the EXIT plane marked with triangles and on the left of the EXIT plane marked with dashed lines in Fig. 6.

Fig. 7 depicts the 3D EXIT charts, when the $x$ axis of Fig. 6 is changed from $I_{E}^{o}(\mathrm{URC})$ to $I_{E}^{i}(\mathrm{URC})$. More explicitly, the vertical EXIT plane seen in Fig. 7, which is independent of the $\mathrm{z}$ axis and the SNR, was computed based on the VL-STC decoder's extrinsic probabilities $L_{e}^{3 \cdot m}(c)$, for $m \in\{1,2,3\}$, at the given values in the $\mathrm{x}$ and $\mathrm{z}$ axes. The slanted EXIT plane shown in Fig. 7 is also SNR-independent and was computed based on the URC decoders' input word extrinsic probabilities $L_{e}^{2 . m}(c)$, for $m \in\{1,2,3\}$, at the given values in the $\mathrm{y}$ and $\mathrm{z}$ axes. The step-wise-linear iterative decoding trajectory displayed in Fig. 7 was plotted based on the extrinsic probabilities $L_{e}^{1 . m}(\mathrm{u}), L_{e}^{2 \cdot m}(c)$ and $L_{e}^{3 \cdot m}(c)$ for $m \in\{1,2,3\}$.

According to [24], the intersection of the planes in Fig. 6 represents the points of convergence between the soft demapper and the URC decoder. The intersection points, where we have $I_{A}(\mathrm{Demod})=I_{E}^{o}(\mathrm{URC})$ at the corresponding $I_{A}^{i}(\mathrm{URC})$ and $I_{A}^{o}(\mathrm{URC})$ values are shown in Fig. 6 as a solid line. The corresponding values of $I_{E}^{i}(\mathrm{URC})$ are shown in Fig. 7 as a solid line on the slanted EXIT plane. Similarly, the intersection of the EXIT planes seen in Fig. 7 represents the points of convergence between the URC decoder and the VL-STC decoder. Hence, by projecting these two intersection curves onto $\mathrm{z}=0$ in Fig. 7 gives us the equivalent 2D EXIT chart seen in Fig. 8. Therefore, the 3D EXIT charts generated for multiple concatenated codes can be projected onto an equivalent 2D EXIT chart [24].

More specifically, we can observe an open tunnel between the EXIT curves of the VL-STC and URC schemes in the 2D EXIT charts of Fig. 8 at $E_{b} / N_{0}=4 \mathrm{~dB}$, which indicates that decoding convergence can be achieved. However, the URC EXIT curve is SNR dependent and by reducing the $E_{b} / N_{0}$ values the angle between the two curves at the topright corner would be further reduced and hence the open tunnel would become closed, hence preventing decoding convergence. Therefore, a better URC code may be designed by ensuring that the EXIT curve exhibits a wider angle with respect to the VL-STC EXIT curve. Note furthermore that the EXIT curve generated for the soft demapper is also depicted in Fig. 8 at $E_{b} / N_{0}=4 \mathrm{~dB}$, where it is flat and it intersects with the VL-STC EXIT curve, before the maximum value of 4.68 bits is reached. Hence, decoding convergence cannot be achieved at $E_{b} / N_{0}=4 \mathrm{~dB}$, when the URC was not invoked between the soft demapper and the VL-STC. Furthermore, at $I_{E}(\mathrm{VL}-\mathrm{STC})=0$ we have $I_{E}^{i}(\mathrm{URC})<I_{E}($ Demod $)$. Hence, before any iteration feedback is exploited, the VL-STCMID scheme would not outperform its VL-STCM counterpart. It was also concluded in [23] that for a three-stage serially concatenated system a unity-rate recursive encoder, such as the URC used, should be employed at the intermediate stage in order to achieve optimal decoding convergence.

Fig. 9 shows the 3D EXIT charts and the corresponding convergence curve of the soft demapper and the URC decoder as well as the actual iterative decoding trajectory for the VL-STCM-ID scheme having $N_{t}=3$ and $N_{r}=2$ when using the correlated source defined in Equation 4. As the source becomes correlated, the entropy of the codeword $c_{m}$ for $m \in\{1,2,3\}$ reduces. Hence, the maximum values for the $\mathrm{x}$ and $y$ axes in Fig. 9 are smaller than those in Fig. 7. However, the open spatial segment of the 3D space between the two EXIT planes becomes wider, when the source is correlated, since the decoders exploit the additional a priori probabilities given by Equation 12. The convergence curve of the soft demapper and the URC decoder is projected as a dashed line onto $I_{E}($ Demod $)=0$ in Fig. 9. Similarly, the projection of the intersection line between the VL-STC and URC EXIT planes is represented by the curve lying on the vertical EXIT plane at $I_{E}($ Demod $)=0$. As can be seen from Fig. 9 at $I_{E}($ Demod $)=0$, an open tunnel exist between the two projection curves at $E_{b} / N_{0}=3 \mathrm{~dB}$. Hence, the iterative decoder converged at $E_{b} / N_{0}=3 \mathrm{~dB}$, i.e. at a $1 \mathrm{~dB}$ lower value, when employing the correlated source instead of the uncorrelated source. Again, the decoding convergence of VL-STCM-ID is limited by the 


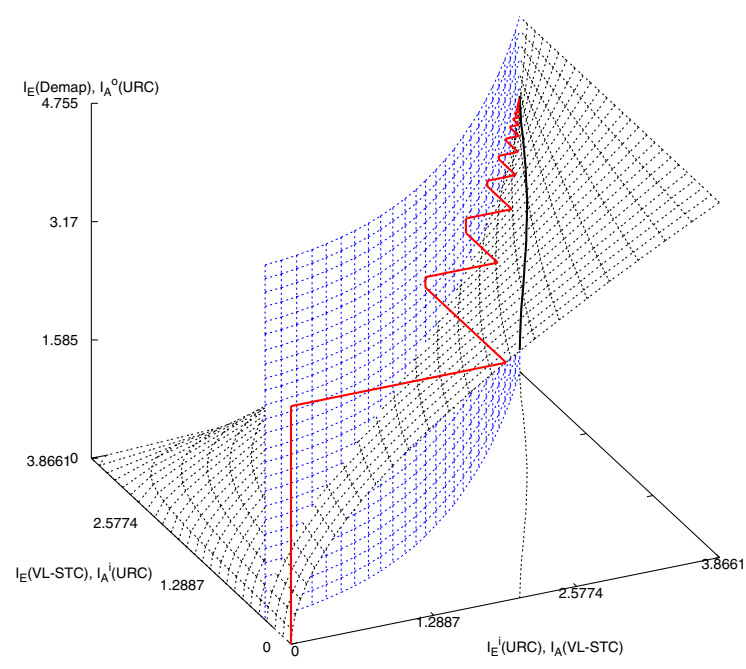

Fig. 9. The 3D EXIT charts for the VL-STCM-ID scheme having $N_{t}=3$ and $N_{r}=2$, when using the correlated source defined in Equation 4 . The iterative trajectory is computed at $E_{b} / N_{0}=3 \mathrm{~dB}$. The maximum value of an axis denotes the entropy of the corresponding symbol.

angle between the two projection curves at the convergence point when using the correlated source. A better URC may be designed for an earlier convergence with the aid of the 3D and 2D EXIT charts, but we leave this issue for future research.

According to the MIMO channel capacity formula derived for the Discrete-Input Continuous-Output Memoryless Channel (DCMC) in [26], the DCMC capacity for the $N_{r}=2$ and $N_{t}=3$ MIMO scheme employing the signal mapper seen in Fig. 1 is $E_{b} / N_{0}=1.25 \mathrm{~dB}$ at a bandwidth efficiency of $3 \mathrm{bit} / \mathrm{s} / \mathrm{Hz}$. Hence, the performance of the VL-STCM-ID scheme is about $2.75 \mathrm{~dB}$ and $1.75 \mathrm{~dB}$ away from the MIMO channel capacity.

\section{Simulation Results}

Let us introduce a Fixed Length (FL) STCM (FL-STCM) scheme as our benchmarker, where the FL Codeword (FLC) matrix is given by:

$$
\mathbf{V}_{F L C}=\left[\begin{array}{llllllll}
0 & 0 & 0 & 0 & 1 & 1 & 1 & 1 \\
0 & 0 & 1 & 1 & 0 & 0 & 1 & 1 \\
0 & 1 & 0 & 1 & 0 & 1 & 0 & 1
\end{array}\right] .
$$

The FL-STCM transmitter obeys the schematic of Fig. 2, except that it employs the $\mathbf{V}_{F L C}$ of Equation 18. For the FLSTCM, the minimum Hamming distance and product distance are 1 and 4, respectively. It attains the same multiplexing gain as that of the VL-STCM or VL-STCM-ID arrangements. Note that it is possible to create an iterative FL-STCM-ID scheme by replacing the VL-STC encoder in Fig. 4 with the FL-STC encoder. However, the EXIT curve of the FL-STC scheme of Equation 18 was found to be too flat for attaining any iteration gain due to its unity minimum Hamming distance. Let us now evaluate the performance of the VL-STCM, VLSTCM-ID and FL-STCM schemes in terms of their source Symbol Error Ratio (SER) versus the $E_{b} / N_{0}$ ratio. Again we

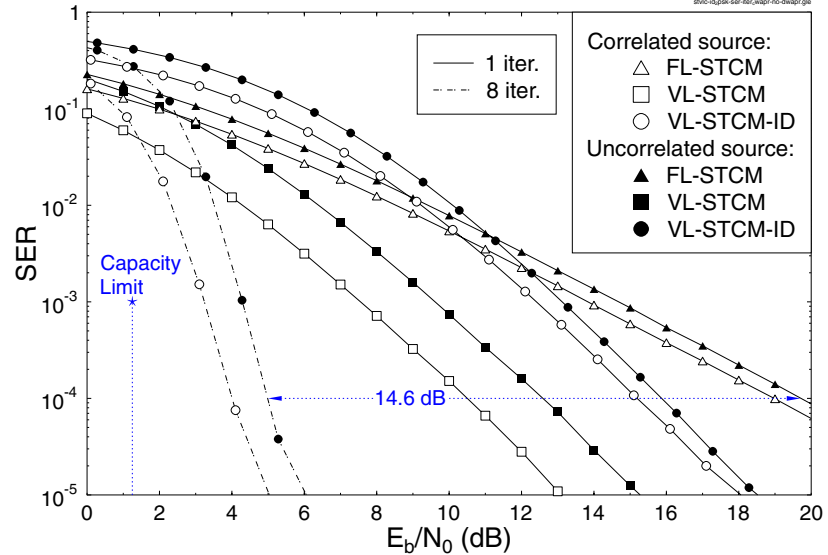

Fig. 10. SER versus $E_{b} / N_{0}$ performance of the VL-STCM, VL-STCMID and FL-STCM schemes, when communicating over uncorrelated Rayleigh fading channels using BPSK, $N_{t}=3$ and $N_{r}=2$.

have $E_{b} / N_{0}=\gamma / \eta$, where $\gamma$ is the SNR per receive antenna and $\eta=\log _{2}\left(N_{s}\right)=3 \mathrm{bit} / \mathrm{s} / \mathrm{Hz}$ is the effective information throughput.

Fig. 10 depicts the SER versus $E_{b} / N_{0}$ performance of the VL-STCM, VL-STCM-ID and FL-STCM schemes, when communicating over uncorrelated Rayleigh fading channels using BPSK, three transmitters, two receivers and a block/interleaver length of 10000 symbols. As expected, the VL-STCM arrangement attains a higher gain, when the source is correlated compared to FL-STCM. However, the FLSTCM benchmarker also benefits from the probability-related a priori information of the source symbols, as the source becomes correlated. By contrast, the coding gain attained as a benefit of transmitting correlated source symbols increases, as the number of iterations invoked by the VL-STCM-ID scheme increases. Although the VL-STCM-ID arrangement performs worse than VL-STCM during the 1st iteration, the performance of VL-STCM-ID at SER $=10^{-4}$ after the 8th iteration is approximately $6.5(15) \mathrm{dB}$ and 7.5 (14.6) $\mathrm{dB}$ better than that of the VL-STCM (FL-STCM) scheme, when employing correlated and uncorrelated sources, respectively. The price of using the VL-STCM-ID scheme for attaining a near-channel-capacity performance is the associated higher decoding and interleaver delay as well as the increased decoding complexity. Hence, for a delay-sensitive and complexityconstrained system using VL-STCM is a better choice. By contrast, for a system that requires a higher performance and can afford a higher delay and complexity, VL-STCM-ID should be employed.

\section{CONCLUSIONS}

An iteratively decoded variable length space-time coded modulation design was proposed. The joint design of sourcecoding, space-time coded modulation and iterative decoding was shown to achieve both spatial diversity and multiplexing gain, as well as coding and iteration gains at the same time. The variable length structure of the individual codewords mapped to the maximum of $N_{t}$ transmit antennas imposes no synchronisation and error propagation problems. The convergence properties of the proposed VL-STCM-ID was analysed 
using 3D symbol-based EXIT charts as well as 2D EXIT chart projections. A significant iteration gain was achieved by the VL-STCM-ID scheme, which hence outperformed both the non-iterative VL-STCM scheme as well as the FLSTCM benchmarker with the aid of $N_{t}$ unity-rate recursive feedback precoders. The VL-STCM-ID scheme attains a near MIMO channel capacity performance. Our future research will incorporate both explicit channel coding and real-time multimedia source codecs.

\section{ACKNOWLEDGEMENTS}

The financial support of both the EPSRC, Swindon UK and the EU under the auspices of the Phoenix and Newcom projects is gratefully acknowledged.

\section{REFERENCES}

[1] C. Shannon, Mathematical Theory of Communication. Champaign, IL: University of Illinois Press, 1963.

[2] G. J. Foschini, Jr. and M. Gans, "On limits of wireless communication in a fading environment when using multiple antennas," IEEE Wireless Pers. Commun., vol. 6, pp. 311-335, Mar. 1998.

[3] E. Telatar, "Capacity of multi-antenna Gaussian channels," European Trans. Telecommun., vol. 10, pp. 585-595, Nov.-Dec. 1999.

[4] G. J. Foschini, Jr., "Layered space-time architecture for wireless communication in a fading environment when using multi-element antennas," Bell Labs Technical J., pp. 41-59, 1996.

[5] V. Tarokh, N. Seshadri, and A. R. Calderbank, "Space-time codes for high rate wireless communication: Performance analysis and code construction," IEEE Trans. Inform. Theory, vol. 44, pp. 744-765, Mar. 1998.

[6] S. Lin, A. Stefanov, and Y. Wang, "Joint source and space-time block coding for MIMO video communications," in IEEE Veh. Technol. Conf., Sep. 2004, pp. 2508-2512.

[7] T. Holliday and A. Goldsmith, "Joint source and channel coding for MIMO systems," in Proc. Allerton Conf. Commun., Control, Comput., Oct. 2004.

[8] S. X. Ng, F. Guo, and L. Hanzo, "Iterative detection of diagonal block space time trellis codes, TCM and reversible variable length codes for transmission over Rayleigh fading channels," in Proc. IEEE Veh. Technol. Conf., Sep. 2004, pp. 1348-1352.

[9] D. Divsalar, S. Dolinar, and F. Pollara, "Serial concatenated trellis coded modulation with rate-1 inner code," in Proc. IEEE GLOBECOM, Nov.Dec. 2000 , pp. $777-782$.

[10] G. Ungerböck, "Channel coding with multilevel/phase signals," IEEE Trans. Inform. Theory, vol. 28, pp. 55-67, Jan. 1982.

[11] A. F. Naguib, V. Tarokh, N. Seshadr, and A. R. Calderbank, "A spacetime coding modem for high-data-rate wireless communications," IEEE J. Select. Areas Commun., vol. 16, pp. 1459-1478, Oct. 1998.

[12] D. Divsalar and M. K. Simon, "Trellis coded modulation for 4800$9600 \mathrm{bits} / \mathrm{s}$ transmission over a fading mobile satellite channel," IEEE J. Select. Areas Commun., vol. 5, pp. 162-175, Feb. 1987.

[13] G. Caire and G. Colavolpe, "On low-complexity space-time coding for quasi-static channels," IEEE Trans. Inform. Theory, vol. 49, pp. 14001416, June 2003.

[14] M. Tao and R. S. Cheng, "Diagonal block space-time code design for diversity and coding advantage over flat Rayleigh fading channels," IEEE Trans. Signal Processing, pp. 1012-1020, Apr. 2004.

[15] T. M. Cover and J. A. Thomas, Elements of Information Theory. New York: John Wiley, IEEE Press, 1991.

[16] G. L. Nemhauser, A. H. G. Rinnooy Kan, and M. J. Todd, Optimization. Amsterdam, The Netherlands: North-Holland, 1989.

[17] L. Hanzo, T. H. Liew, and B. L. Yeap, Turbo Coding, Turbo Equalisation and Space Time Coding for Transmission over Wireless channels. New York: John Wiley, IEEE Press, 2002.

[18] X. Li and J. A. Ritcey, "Bit-interleaved coded modulation with iterative decoding using soft feedback," IEE Electron. Lett., vol. 34, pp. 942-943, May 1998

[19] P. Robertson, E. Villebrun, and P. Höher, "A comparison of optimal and sub-optimal MAP decoding algorithms operating in log domain," in Proc. IEEE International Conf. Commun., pp. 1009-1013, June 1995.
[20] S. ten Brink, "Convergence behaviour of iteratively decoded parallel concatenated codes," IEEE Trans. Commun., vol. 49, pp. 1727-1737, Oct. 2001.

[21] H. Chen and A. Haimovich, "EXIT charts for turbo trellis-coded modulation," IEEE Commun. Lett., vol. 8, pp. 668-670, Nov. 2004.

[22] A. Grant, "Convergence of non-binary iterative decoding," in Proc. IEEE GLOBECOM, Nov. 2001, pp. 1058-1062.

[23] M. Tuchler, "Convergence prediction for iterative decoding of threefold concatenated systems," in Proc. IEEE GLOBECOM, Nov. 2002, pp. $1358-1362$.

[24] F. Brannstrom, L. K. Rasmussen, and A. J. Grant, "Convergence analysis and optimal scheduling for multiple concatenated codes," IEEE Trans. Inform. Theory, pp. 3354-3364, Sep. 2005.

[25] J. Kliewer, S. X. Ng, and L. Hanzo, "On the computation of EXIT characteristics for symbol-based iterative decoding," in Proc. 4th International Symposium Turbo Codes with 6th International ITG-Confe. Source Channel Coding, Apr. 2006.

[26] S. X. Ng and Hanzo, "On the MIMO channel capacity of multidimensional signal sets," IEEE Trans. Veh. Technol., vol. 55, no. 2, pp. 528-536, Mar. 2006

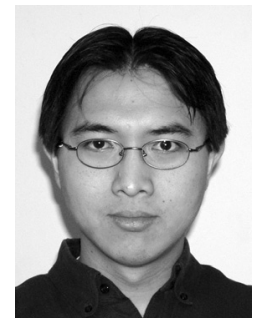

Soon Xin Ng (S'99-M'03) received the B.Eng. degree (First class) in electronics engineering and the $\mathrm{Ph} . \mathrm{D}$. degree in mobile communications from the University of Southampton, Southampton, U.K., in 1999 and 2002, respectively.

Currently, he is continuing his research as a Postdoctoral Research Fellow at the University of Southampton. His research interests are mainly in adaptive coded modulation, channel coding, turbo coding, space-time coding, and joint source and channel coding. He has published numerous papers and coauthored a book in this field.

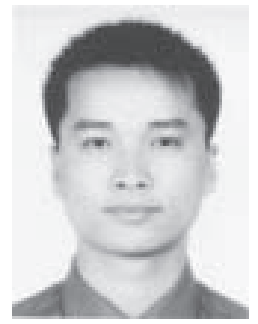

Jin Wang ( $\left.\mathrm{S}^{\prime} 03\right)$ received the B.E. degree from University of Science and Technology of China (USTC), Hefei, China in 1999 and the M.E. degree in video signal processing from Graduate School of Chinese Academy of Sciences (GSCAS), Beijing, China, in 2002.

$\mathrm{He}$ is currently pursuing his $\mathrm{PhD}$ degree with the Communications Research Group at the School of ECS, University of Southampton, Southampton, UK. His research interests include video coding, channel coding, joint source/channel coding, and iterative detection and decoding for digital communication systems.

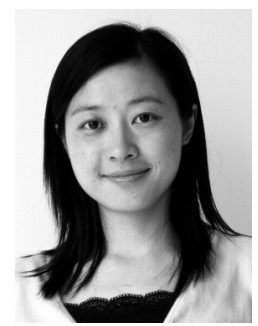

Meixia Tao (S'00-M'04) received the B.S. degree in Electronic Engineering from Fudan University, Shanghai, China, in 1999, and the Ph.D. degree in Electrical and Electronic Engineering from Hong Kong University of Science \& Technology in 2003.

From Aug. 2003 to Aug. 2004, she was a Member of Professional Staff in the Wireless Access Group at Hong Kong Applied Science \& Technology Research Institute Co. Ltd. Since Aug 2004, she has been with the Dept. of Electrical and Computer Engineering at National University of Singapore. Her research interests include multiple antenna techniques, coding and modulation, dynamic resource allocation in wireless networks, and cross-layer design and optimization. 


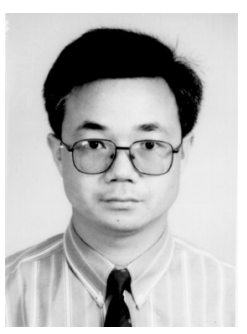

Lie-Liang Yang (M'99-SM'03) received his B.Eng. degree in communication engineering from Shanghai TieDao University, Shanghai, China in 1988, and his M.Eng, Ph.D. degrees in communications and electronics from Northern Jiaotong University, Beijing, China in 1991 and 1997, respectively. From June 1997 to December 1997 he was a visiting scientist of the Institute of Radio Engineering and Electronics, Academy of Sciences of the Czech Republic. Since December 1997, he has been with the Communications Research Group, School of Electronics and Computer Science, University of Southampton, U.K, where he was first a Postdoctoral Research Fellow (Dec. 1997 - Aug. 2002), then a Lecturer (Sept. 2002 - Feb. 2006), and currently holds the academic post of Readership. Dr. Yang's research has covered a wide range of areas in telecommunications, which include error control coding, modulation and demodulation, spreadspectrum communications and multiuser detection, synchronization, spacetime processing, adaptive wireless systems, as well as wideband, broadband and ultrawide-band code-division multiple-access (CDMA). He has published over 130 papers in journals and conference proceedings, coauthored one book and published several book chapters. He was awarded the Royal Society SinoBritish Fellowship in 1997 and the EPSRC Research Fellowship in 1998. Dr. Yang is currently an associate editor for both the Journal of Communications and Networks (JCN) and the Journal of Communications (JCM).

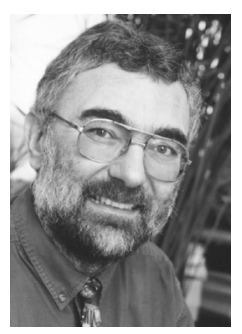

Lajos Hanzo (M'91-SM'92-F'04) Fellow of the Royal Academy of Engineering, received his firstclass degree in electronics in 1976 and his doctorate in 1983. In 2004 he was awarded the Doctor of Sciences (DSc) degree by the University of Southampton, UK. During his career in telecommunications he has held various research and academic posts in Hungary, Germany and the UK. Since 1986 he has been with the Department of Electronics and Computer Science, University of Southampton, UK, where he holds the chair in telecommunications. He has co-authored 12 books, totalling 9000 pages on mobile radio communications, published in excess of 600 research papers, has acted as TPC Chair of numerous major IEE and IEEE conferences, presented various keynote lectures and has been awarded a number of distinctions. Currently he heads an academic research team, working on a range of research projects in the field of wireless multimedia communications sponsored by industry, the Engineering and Physical Sciences Research Council (EPSRC) UK, the European IST Programme and the Mobile Virtual Centre of Excellence (VCE), UK. He is an enthusiastic supporter of industrial and academic liaison and he offers a range of industrial courses. Lajos is also an IEEE Distinguished Lecturer of both the Communications as well as the Vehicular Technology Society, a Fellow of both the IEEE and the IEE. He is an editorial board member of the Proceedings of THe IEEE and a Governer of the IEEE VT Society. For further information on research in progress and associated publications, please refer to http://www-mobile.ecs.soton.ac.uk 